\title{
Gold nanoparticle-coated biomaterial as SERS micro-probes
}

\author{
G V PAVAN KUMAR \\ Division of Physics and Chemistry, Indian Institute of Science Education and Research (IISER), Pune 411 021, India
}

MS received 18 September 2010; revised 28 October 2010

\begin{abstract}
We report for the first time, on the utility of plant-based biomaterial as enhanced-Raman scattering probes. The bio-substrate used in this study are commonly found in plant extracts, and are cost-effective, mechanically robust, flexible and easily transportable. The probe was fabricated by coating the plant extract with gold nanoparticles and characterized. By employing a 'single-touch contact' method, we reveal the ability of these probes to detect routinely used Raman markers such as 2-napthalenethiol and rhodamine $B$, at nano-molar concentrations, in dry and liquid forms, respectively. Reproducibility of the signals with variation $<5 \%$, and the ability to detect biomolecules are demonstrated herein. We envision these bio-probes as potential candidates for enhanced Raman sensing in chemical, environmental, and archaeological applications. By further engineering the shape, morphology, and surface chemistry of these micro-probes, we foresee their utility as miniaturized, natural SERS substrates.
\end{abstract}

Keywords. Surface enhanced Raman scattering; micro-probes; biomaterial; plasmonics.

\section{Introduction}

When a molecule is placed either in the vicinity or on the surface of a nanoscale plasmonic material, such as gold or silver nanoparticle, the Raman scattering intensity of the molecule is enhanced by many orders of magnitude. This phenomenon is called as surface enhanced Raman scattering (SERS) (Moskovits 1985), and in recent times, has emerged as a chemical imaging tool with single molecule/particle sensitivity (Kneipp et al 2006; Lal et al 2007; Willets and Van Duyne 2007). Electromagnetic and chemical enhancement mechanisms explain the observed increment in the intensity of the Raman scattered signal (Moskovits 1985; Aroca 2006; Le Ru and Etchegoin 2009). By harnessing these mechanisms, SERS has been utilized for various applications in chemistry, physics, biology and engineering (Haes and Van Duyne 2002; West and Halas 2003; Jackson and Halas 2004; Lal et al 2007; Willets and Van Duyne 2007). Although chemical detection using surface enhanced Raman spectroscopy has achieved a fair degree of success, there is a need to prepare appropriate substrates which are robust, reproducible, portable, adaptable and inexpensive. Added to this, if enhanced Raman scattering probes need to be utilized for special applications such as forensic and archaeological sciences, then their design should account for the measurement options on-site (for example, blood stain, pigment of a painting), and the chemical information captured by the probe should be easily retrievable upon analysis in the laboratory. Furthermore, it is also imperative to explore

(pavan@iiserpune.ac.in) various unconventional, miniaturized materials as enhanced Raman scattering probes that have the capability to (i) interact with a sample for a short period of time, (ii) capture the chemical signature on the surface of the probe, (iii) detach from the sample surface, and (iv) transportable to the laboratory for further analysis. Till date, a variety of SERS substrates have been produced both via top-down fabrication methods (Claro and Rojas 1993; Liu et al 2006; Cui et al 2008; De Angelis et al 2008; Andrea et al 2009; Bhuvana and Kulkarni 2009; Linn et al 2009), and bottom-up self assembly approach (Jensen et al 2000; Haynes and Van Duyne 2001; Jackson and Halas 2004; Whitney et al 2007; Le et al 2008). However, there are a very few efforts, that have utilized biomaterials as SERS substrates, such as the usage of chitinous nanostructures on the wings of butterfly (Garrett et al 2009). Also, to our knowledge, microstructures of plants have not been utilized as SERS substrates.

In the hindsight of these issues, herein we report a new route towards fabrication of sharp, metal nanoparticle-coated probe by utilizing the sub-micron features of Arabidopsis trichomes, a small flowering plant in the mustard family. Arabidopsis is a model plant species that is desirable for plant science research because of its short life cycle, small size, diploid genome, and availability of sophisticated genomic resources that have been created by an international research community. One aspect of Arabidopsis biology is the use of the leaf hair cells (trichomes) as a cell type to study the cytoskeleton basis of plant morphogenesis (Smith and Oppenheimer 2005). The rationale behind choosing this substrate is that plant-based biomaterials have evolved with unusual micro and nanostructures that have sophisticated optical properties which can be harnessed as optical probes (Lee 2009). Inspired by this knowledge, we 
have explored the possibility of utilizing plant trichomes as SERS microprobes.

In the current work, we use leaf trichomes as a biological material-template for the generation of a new type of enhanced Raman scattering probe. We reveal the preparation of a highly sensitive probe that can interact with a sample, and capture its chemical information in a single touch. These substrates can be detached from the sample after interaction, and be used for further Raman analysis. The main objectives of this study were to (a) isolate the sharply pointed plantcells (SPPC) from Arabidopsis leaves and coat them with Au nanoparticles, (b) characterize the Au nanoparticle coated micro-probes, and (c) test their utility as SERS probes. Our main aim is to show that micro-scale plant-based biomaterial can serve as miniaturized SERS substrates. Another attractive feature of our demonstration is the fact that the probes proposed are naturally occurring biological materials as opposed to the conventional lithography-based sharp structures, which make them inexpensive and readily usable.

\section{Experimental}

\subsection{Extraction of tips}

For all the experiments, Arabidopsis thaliana Col-0 background was used as the wild type. These relevant structures were from the plants of mustard family and were extracted on the hills of Panchavati, Pune, India.

Trichomes first emerge from the epidermis as a small cylindrical projection that elaborates 3 or 4 bulges at an early developmental stage (figure 1a). Subsequently these cells expand greatly and the branch tip is refined from a blunt tip to a sharp structure with a radius of curvature of $\sim 400 \mathrm{~nm}$ (Szymanski et al 1998). Figure 1a shows SEM image of a typical trichome on a leaf. Sharply pointed plant cell (SPPC) structures are evident on the surface of a leaf. The SPPCs taper towards a sharp apex and length of these tips can be as long as $100-150 \mu \mathrm{m}$. Figure $1 \mathrm{~b}$ shows an optical transmission image of trichomes on the surface of a leaf. These trichomes are abundant on the leaves and can be easily visualized using an optical microscope. Figure 1c shows a typical optical transmission microscopy image of single SPPC. Most of the cells are sharp, and exhibit similarities in their morphology. It is to be noted that the apex of the tip can be very sharp (300-400 $\mathrm{nm})$.

Figure 2 shows schematic of a one-touch contactenhanced Raman probing methodology by harnessing SPPC of Arabidopsis plant. Leaves containing trichomes are detached from this plant with the aid of a dissection microscope. Unicellular trichomes are branched, with each branch yielding an extremely sharp tip. The trichomes are plucked from the leaf surface and immersed in a container of gold nanoparticles. After $60 \mathrm{~min}$, the trichomes are removed from the nanoparticle solution, and the tip is brought in contact with an analyte (solid or liquid) to be interrogated. After a few seconds, the trichome is detached from the sample surface, and further used for Raman spectroscopy analysis. All chemicals were purchased from Sigma Aldrich (USA). FE-SEM images were acquired using Hitachi S-4800 Field Emission SEM.

Surface enhanced Raman spectroscopy measurements were performed using Lab-Ram HR Jobin Yvon in backscattering geometry. A high numerical aperture, long working
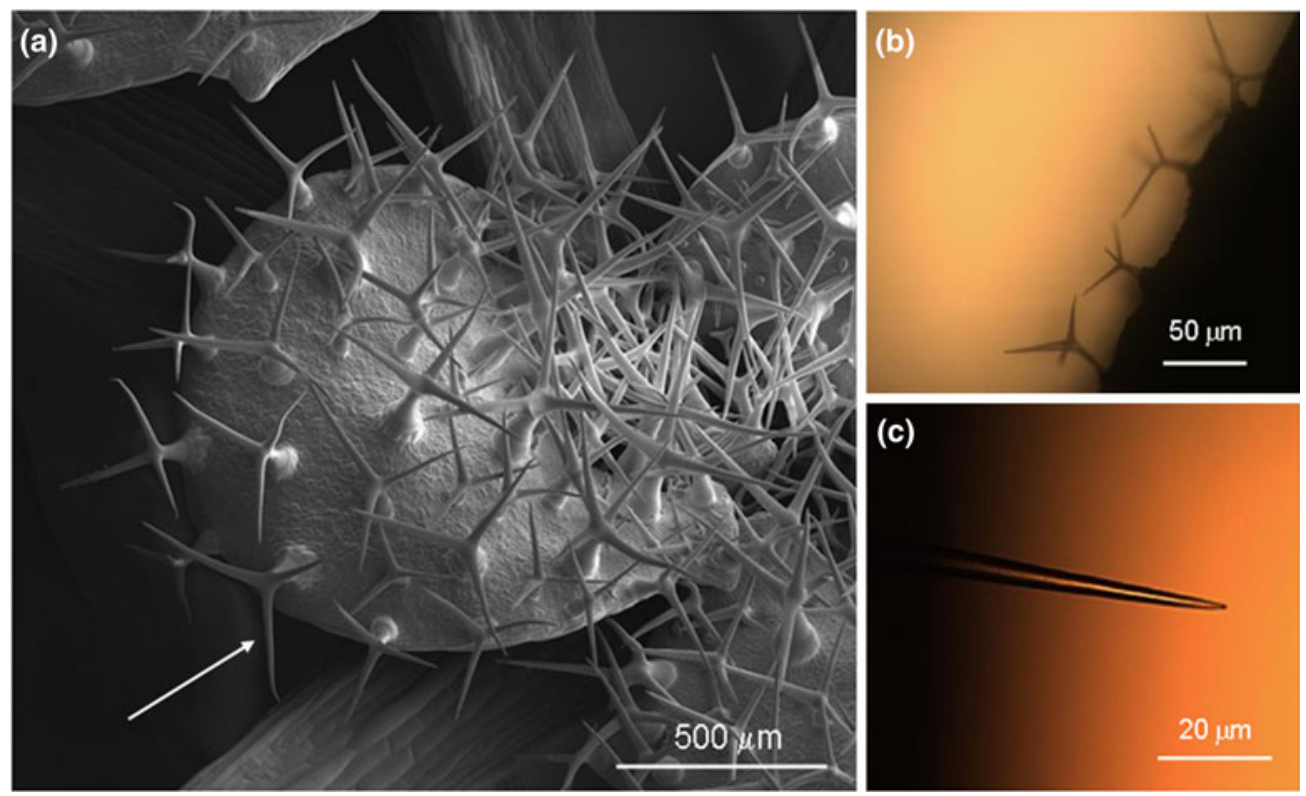

Figure 1. (a) SEM image of Arabidopsis trichomes, (b) optical transmission microscopy image of the trichomes and (c) optical transmission microscopy image of an isolated tip from plant leaf. The arrow indicates an individual cell. 


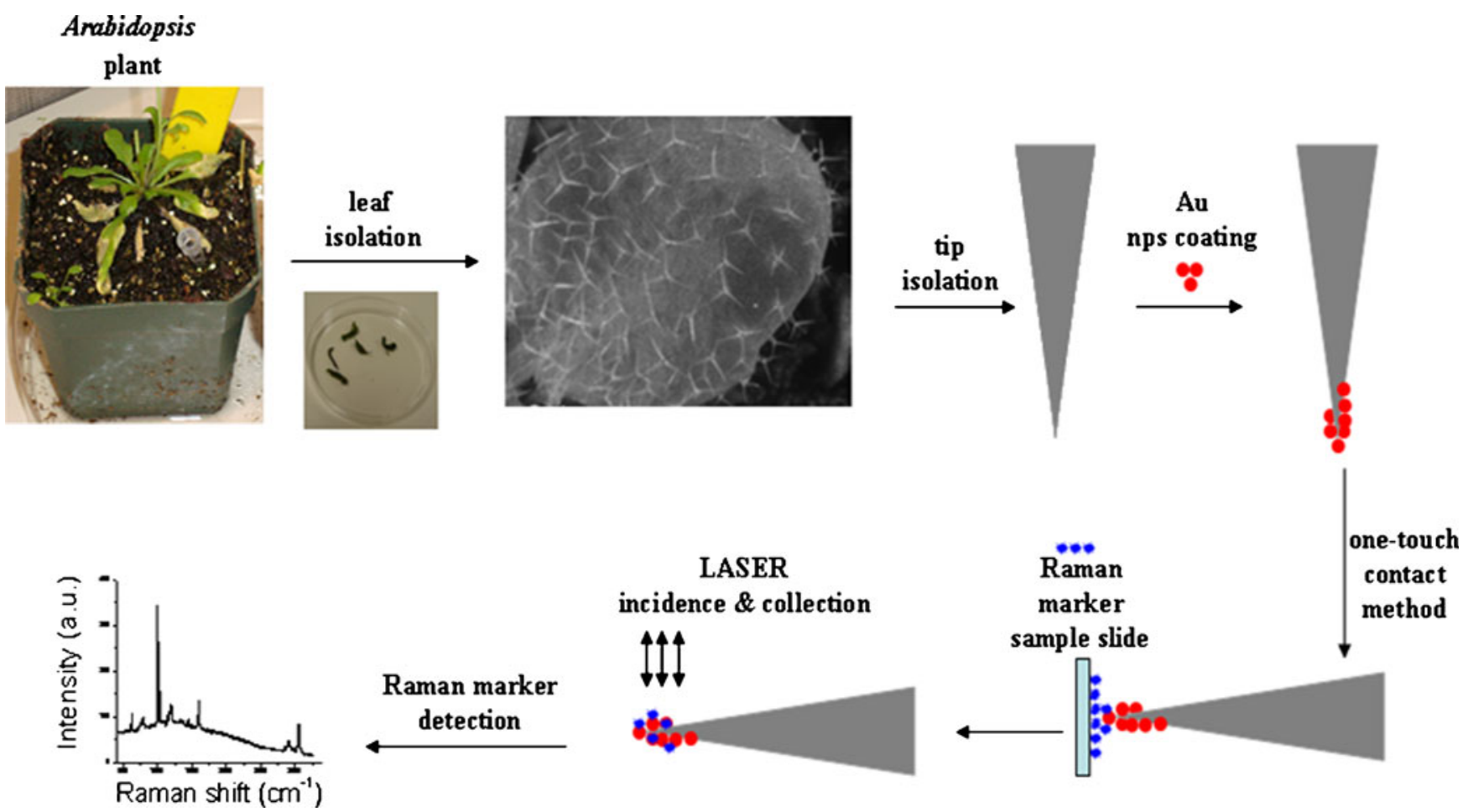

Figure 2. Schematic of the one-touch contact-enhanced Raman probing methodology by harnessing sharply pointed plant cells of Arabidopsis trichomes.

distance objective lens $(50 \times, 0 \cdot 5 \mathrm{NA})$ was employed for illumination and collection of laser light. For the present work, we utilized the $633 \mathrm{~nm}$ laser as the excitation source and the spectra were recorded at a resolution of $\sim 2 \mathrm{~cm}^{-1}$. Optical images were captured with the camera attached to the Raman microscope.

\section{Results and discussion}

\subsection{Au nanoparticle coating and characterization of SPPC}

Citrate reduced $\mathrm{Au}$ nanoparticles were produced by Lee and Meisel method. Further details on nanoparticle synthesis can be found in $\S S 1$ of supporting information. After isolating the tips from the surface of the leaves, they were first silane functionalized and immersed in citrate-reduced Au nanoparticle (Au nps) solution for $60 \mathrm{~min}$, followed by drying at room temperature. We observed that an interaction time of 60 min was optimum for uniform coating of nanoparticles, and a longer time scale resulted in aggregation of nanoparticles. The silane coating facilitated chemical functionalization that helps in specific binding of Au nps to the tip. This procedure helped in achieving a uniform coating of nanoparticles around the apex of the tip due to covalent functionalization. Figure 3 shows a typical SPPC functionalized with Au nanoparticles. Our aim was to coat the apex of the tip with Au nps, which can act as the Raman enhancing platform on which molecules can reside. The FE-SEM image of a SPPC

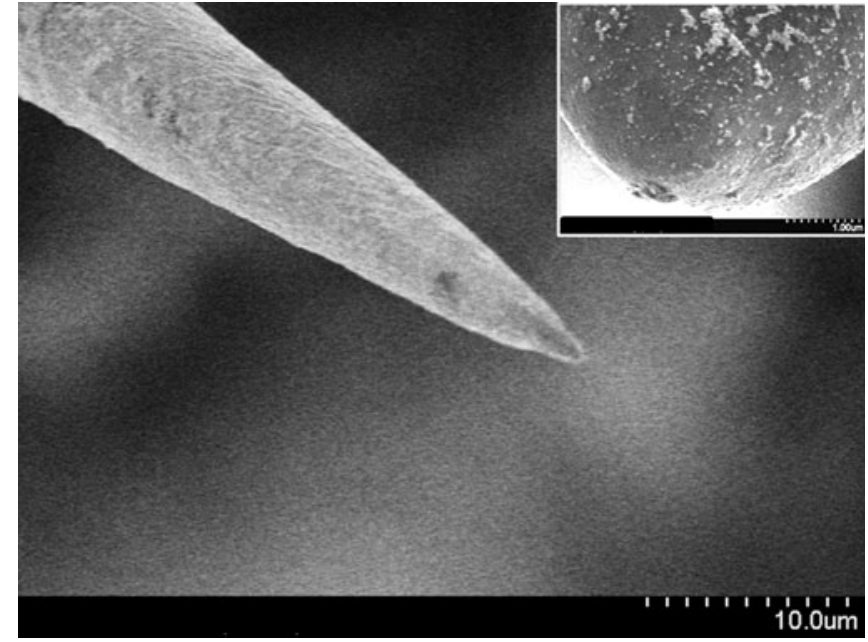

Figure 3. FE-SEM image of a sharply pointed plant cell coated with Au nanoparticles. The inset shows distribution of Au nanoparticles on the apex of the tip.

coated with Au nps (inset in figure 3) shows distribution of Au nanoparticles on the surface of SPPC with nanoscale corrugation ideally suited for SERS. Typically, the size of the apex of the tip was observed to be in the range between 200 and $400 \mathrm{~nm}$ and can be further lessened by employing techniques such as e-beam lithography and focused ion-beam milling. 


\subsection{Au nps coated sharply pointed cell as enhanced Raman scattering probes}

3.2a One-touch contact method: To apply enhancedRaman scattering techniques in areas such as forensic and archaeological sciences, it is always desirable to have a substrate that can be easily transportable from the sample-site to the laboratory. Also, interaction between the Raman probe and the sample should be confined to limited space and time. In order to address this issue, we employ the 'one-touch contact' method. In this method, first, Au nps coated SPPC trichomes are microscopically attached to a sharp syringe needle for easy handling of the tip. Next, the interaction between the sample and the tip was constrained to a single, gentle, touch of the probe on the surface of the sample. The contact time between the sample and the tip was limited to a few seconds, after which, the tip was retrieved away from the surface of the sample. It is to be noted that the SPPCs are elastic in nature and therefore, flexible. We observed that the tips were extremely robust to soft contacts, and retained their shape and morphology even after its interaction ( $\$$ S2 of supporting information) with the sample.

3.2b Raman probing of the tips: After the one-touch contact method, the syringe needle attached to the trichome was transferred to the stage of Raman microscopy. The laser spot was precisely focused on to the apex of the tip. After achieving this, Raman scattering measurement can be performed, either in the back-scattering or inverted geometry mode. Figure 4a shows an enhanced Raman spectrum captured from the apex of a tip (inset of figure 4a) that was previously in 'one-touch contact' with a cover-slip drop-casted with 2napthalenethiol solution $(100 \mathrm{nM})$. A spectrum with a high signal-to-noise ratio of naphthalenethiol, with a small background signal could be obtained from this set up. Control experiments constitute Raman spectra from bare, uncoated tip and Au nps-coated tip without the target analyte, as presented in $\S S 3$ of the supporting information. The approximate enhancement factor calculated for these tips was found to be $3.22 \times 10^{6}$ ( $\$ \mathrm{~S} 4$ in supporting information). This enhancement factor was comparable (table S1 in supporting information) to other conventional $\mathrm{Au}$ substrates that were produced via relatively expensive techniques. We observe that the enhancement factor of plant substrates was on par with fabricated substrates.

3.2c Utility in liquids: We demonstrate the robustness of the probe in a liquid environment, by intentionally pointing the $\mathrm{Au}$ coated SPPC into a droplet of $1 \mathrm{nM}$ rhodamine $\mathrm{B}$ solution (inset of figure $4 \mathrm{~b}$ ). With the laser focused on the apex of the tip, Raman spectrum was captured. In figure $4 \mathrm{~b}$ we show the Raman spectra captured from the apex of the tip for an accumulation time of $1 \mathrm{~s}$. The high signal-to-noise ratio spectrum observed over a broad background of the tip shows that our approach can be harnessed for liquid-phase Raman sensing.
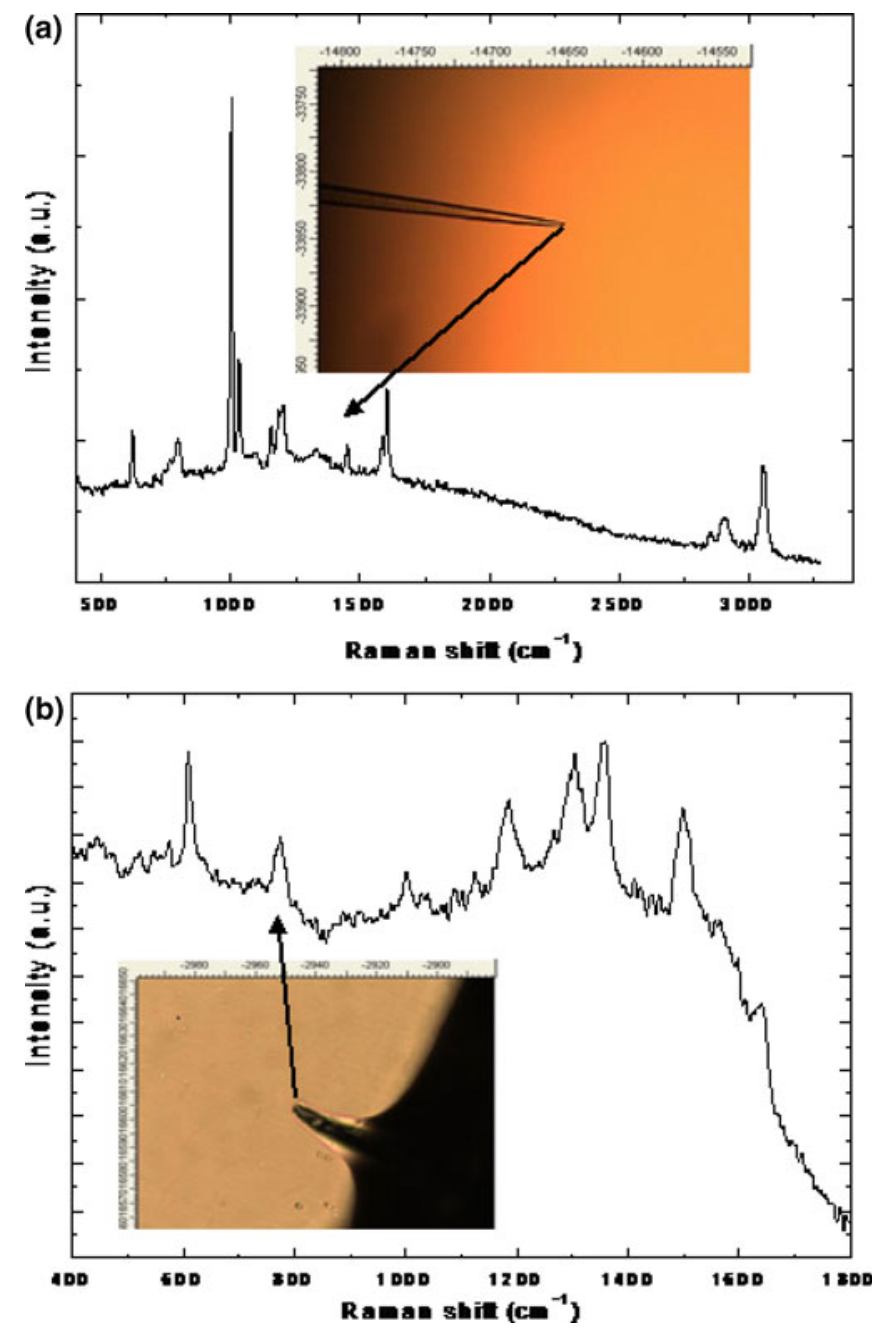

Figure 4. (a) Enhanced Raman spectra of 2-napthalenethiol captured at the apex of the tip shown in the inset. Before capturing the spectra, the tip was brought in contact with a cover-slip drop-casted with 2-napthalenethiol (100 $\mathrm{nM}$ concentration) and (b) enhanced Raman spectra of rhodamine B (1 nM concentration) captured using $\mathrm{Au}$ nanoparticle coated tip. The insets in (a) and (b) are optical images of the tip.

3.2d Reproducibility: In order to confirm the consistency of our one-touch contact method, several tips were tested and the results from four such measurements are provided. The test molecule used was rhodamine $6 \mathrm{G}(1 \mu \mathrm{M})$ and measurements were performed with a $633 \mathrm{~nm}$ laser, at $5 \mathrm{~mW}$ power with $40 \mathrm{~s}$ accumulation. Figure 5 shows the optical image of the four different tips used in our study, accompanied by their respective SERS signals, showing a high degree of consistency and demonstrating good reproducibility of our method. The variation in the Raman signal intensity from five different tips was $<5 \%$.

3.2e Utility of SPPC in detection of biomolecules: In order to show the potential in biological applications, adenine, adenosine-tri-phosphate (ATP), TAT peptide and BSA 


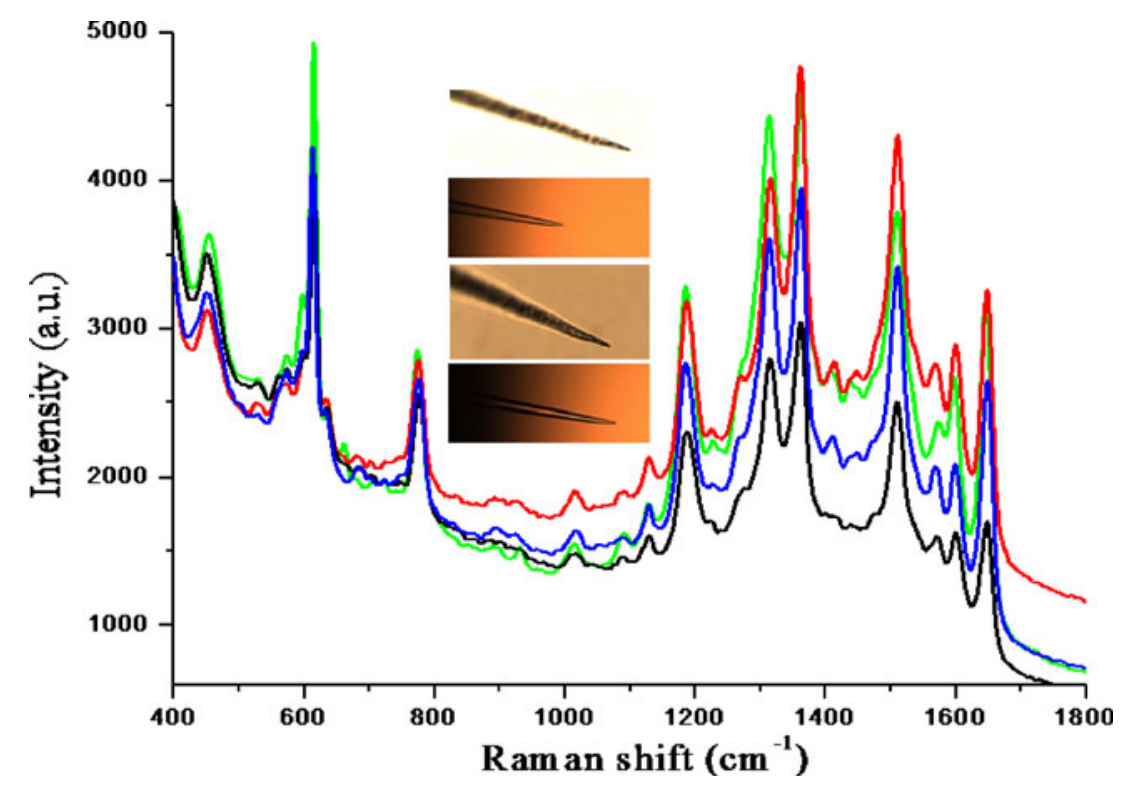

Figure 5. Reproducibility of SERS signals with 4 different tips shown in the inset. The test molecule was rhodamine $6 \mathrm{G}(1 \mu \mathrm{M})$. The inset shows the optical transmission microscopy image of the tips. All 4 measurements were performed under the same experimental conditions.

protein were interrogated. All the samples were kept at the same concentration $(1.5 \mu \mathrm{M})$ and experiments were performed with the same parameters as discussed before. Section S5 in supporting information shows the SERS spectra obtained by one-touch contact method. We observed a high signal-to-noise and the obtained spectra were in agreement with the positive control experiments (with $\mathrm{Au}$ nanoparticles). This clearly indicates the potential of these miniaturized tips for high fidelity biomolecular detection.

\section{Conclusions}

To summarize, we show the utility of trichomes of Arabidopsis plant as enhanced Raman scattering probes. By employing a 'one-touch contact' method, we capture the chemical information from a molecular surface, and retrieve it by performing Raman scattering experiments. The feasibility of utilizing plant-based tips in liquid environment was revealed by performing Raman scattering measurements in rhodamine B solution at sub-nanomolar concentrations as well as in interrogating peptides and proteins. High signal-to-noise ratio was observed for all the cases. As a future prospect, these plant-based tips can be harnessed for applications in chemical, forensic and archaeological sciences, where robust, transportable SERS probes are necessary.

\section{Acknowledgements}

The author thanks Prof. J Irudayaraj and Prof. D Szymanski for their feedback and plant facilities during the initial stage of the project. The author is also thankful to IISER, Pune and IIT, Bombay for experimental facilities. DST, India is acknowledged for DST Nanoscience project at IISER, Pune and a DST Ramanujan fellowship to the author.

\section{Supporting information}

Experimental details of the synthesis of $\mathrm{Au}$ nanoparticles, Raman scattering control experiments, test of robustness, enhancement factor calculation and tabulation, and control experiments on cells can be viewed in supplementary information.

\section{References}

Andrea C D, Neri F, Ossi P M, Santo N and Trusso S 2009 Nanotechnology 20245606

Aroca R 2006 Surface-enhanced vibrational spectroscopy (Chichester, UK: John Wiley \& Sons)

Bhuvana T and Kulkarni G U 2009 Nanotechnology 20045504

Claro F and Rojas R 1993 J. Phys.: Condens. Matter 5 A187

Cui B, Clime L, Li K and Veres T 2008 Nanotechnology 19 145302

De Angelis F, Patrini M, Das G, Maksymov I, Galli M, Businaro L, Andreani L C and Di Fabrizio E 2008 Nano Lett. 82321

Garrett N L, Vukusic P, Ogrin F, Sirotkin E, Winlove C P and Moger J 2009 J. Biophoton. 2157

Haes A J and Van Duyne R P 2002 J. Am. Chem. Soc. 12410596

Haynes C L and Van Duyne R P 2001 J. Phys. Chem. B105 5599

Jackson J B and Halas N J 2004 Proc. Nat. Acad. Sci. USA 101 17930 
Jensen T R, Malinsky M D, Haynes C L and Van Duyne R P 2000 J. Phys. Chem. B104 10549

Kneipp K, Moskovits M and Kneipp H (eds) 2006 Surfaceenhanced Raman scattering: Physics and applications (Berlin, Heidelberg: Springer-Verlag)

Lal S, Link S and Halas N J 2007 Nature Photonics 1641

Le Ru E C and Etchegoin P G 2009 Principles of surface-enhanced Raman spectroscopy and related plasmonic effects (Amsterdam: Elsevier)

Le F, Brandl D W, Urzhumov Y A, Wang H, Kundu J, Halas N J, Aizpurua J and Nordlander P 2008 Acs Nano 2707

Lee D W 2009 Plant tissue optics: micro- and nanostructures. Biomimetics and Bioinspiration (San Diego, CA: SPIE) p. 740104
Linn N C, Sun C-H, Arya A, Jiang P and Jiang B 2009 Nanotechnology 20225303

Liu Y J, Fan J G, Zhao Y P, Shanmukh S and Dluhy R A 2006 Appl. Phys. Lett. 89173134

Moskovits M 1985 Rev. Mod. Phys. 57783

Smith L G and Oppenheimer D G 2005 Ann. Rev. Cell Dev. Bio. 21271

Szymanski D B, Jilk R A, Pollock S M and Marks M D 1998 Development 1251161

West J L and Halas N J 2003 Ann. Rev. Biomed. Eng. 5285

Whitney A V, Elam J W, Stair P C and Van Duyne R P 2007 J. Phys. Chem. C111 16827

Willets K A and Van Duyne R P 2007 Ann. Rev. Phys. Chem. $\mathbf{5 8} 267$ 